\title{
An Algorithm for Determining Invertible Quadratic Isoparametric Finite Element Transformations
}

\author{
By David A. Field
}

\begin{abstract}
This paper derives an algorithm which determines the invertibility of arbitrary two-dimensional quadratic isoparametric finite element transformations. Theorems verifying the algorithm and guiding the construction of invertible transformations are proven.
\end{abstract}

1. Introduction. The use of the finite element method for solving boundary value problems requires that the Jacobians of the associated finite element transformations do not change sign. Indeed, the inversion of these Jacobians is necessary to obtain numerical values such as stress at prescribed points.

In this journal [1], Frey, Hall, and Porsching's paper concerned the construction and the numerical inversion of 8-node two-dimensional quadratic isoparametric transformations by restricting the locations of the 8-nodes and by the assumption of a boundary hypothesis. Ensuring that the Jacobians of such transformations did not vanish was a substantial difficulty.

The major contribution of this paper is the derivation of an algorithm which determines whether an arbitrary 8-node two-dimensional quadratic isoparametric transformation has a vanishing Jacobian. This paper also includes an algorithm for constructing invertible transformations which are more flexible than those found in [1]. Both algorithms are based upon interpreting the Jacobian as a two-dimensional surface.

2. Verification of Invertibility. Let the 8-node two-dimensional quadratic isoparametric transformation $T: U=[0,1] \times[0,1] \rightarrow \mathbf{R}^{2}$ be defined by

$$
\begin{aligned}
T(r, s)= & (1-r) \bar{x}(0, s)+r \bar{x}(1, s)+(1-s) \bar{x}(r, 0) \\
& +s \bar{x}(r, 1)-(1-s)(1-r) \bar{x}(0,0)-(1-s) r \bar{x}(1,0) \\
& -s(1-r) \bar{x}(0,1)-s r \bar{x}(1,1),
\end{aligned}
$$

where for $\phi_{1}(\tau)=(2 \tau-1)(\tau-1), \phi_{2}(\tau)=4 \tau(1-\tau)$, and $\phi_{3}(\tau)=(2 \tau-1) \tau$,

$$
\begin{aligned}
& \bar{x}(0, s)=\phi_{1}(s) \bar{x}(0,0)+\phi_{2}(s) \bar{x}\left(0, \frac{1}{2}\right)+\phi_{3}(s) \bar{x}(0,1), \\
& \bar{x}(1, s)=\phi_{1}(s) \bar{x}(1,0)+\phi_{2}(s) \bar{x}\left(1, \frac{1}{2}\right)+\phi_{3}(s) \bar{x}(1,1), \\
& \bar{x}(r, 0)=\phi_{1}(r) \bar{x}(0,0)+\phi_{2}(r) \bar{x}\left(\frac{1}{2}, 0\right)+\phi_{3}(r) \bar{x}(1,0), \\
& \bar{x}(r, 1)=\phi_{1}(r) \bar{x}(0,1)+\phi_{2}(r) \bar{x}\left(\frac{1}{2}, 1\right)+\phi_{3}(r) \bar{x}(1,1),
\end{aligned}
$$

Received July 31, 1980; revised March 12, 1981.

1980 Mathematics Subject Classification. Primary 65N30; Secondary 65H10. 
and

$$
T(r, s) \equiv\left(\begin{array}{l}
x(r, s) \\
y(r, s)
\end{array}\right) \equiv \bar{x}(r, s) .
$$

Sufficient conditions for $T$ to be invertible are given by the following theorem, which is a restatement of a theorem of de la Vallée Poussin [4, pp. 283-284], [2, p. 252].

THEOREM 2.1. If the Jacobian of $T$ does not vanish on $U$ and $T$ is one-to-one on the boundary of $U$, then $T$ is a bijection on $U$.

Verifying that the images under $T$ of adjacent edges of the boundary of the square $U$ intersect only at the specified corner node is a straightforward process of solving simultaneous equations. That the images under $T$ of opposite edges of $U$ do not intersect can be verified by showing that the Jacobian of $T$ does not vanish on $U$. Thus, after verifying that $T$ is one-to-one on pairs of adjacent edges of $U, T$ is invertible whenever it can be shown that the Jacobian, $J$, of $T$ does not vanish on $U$.

The remainder of this section develops an algorithm to detect whether the Jacobian of $T$ vanishes on $U$.

In order to express the Jacobian of $T$, the following notation will be convenient:

$$
\bar{x}(r, s) \equiv \bar{x}_{r s} \equiv\left(\begin{array}{l}
x_{r s} \\
y_{r s}
\end{array}\right) ; \quad \bar{x}_{\alpha s} \equiv \bar{x}_{1 s}-\bar{x}_{0 s} ; \quad \bar{x}_{r \beta} \equiv \bar{x}_{r 1}-\bar{x}_{r 0}
$$

and

$$
\Delta \bar{x}_{r \frac{1}{2}}=\bar{x}_{r 0}-2 \bar{x}_{r \frac{1}{2}}+\bar{x}_{r 1}, \quad \Delta \bar{x}_{\frac{1}{2} s} \equiv \bar{x}_{0 s}-2 \bar{x}_{\frac{1}{2} s}-\bar{x}_{1 s}
$$

Thus

$$
\bar{x}_{\alpha \beta}=\bar{x}_{\alpha 1}-\bar{x}_{\alpha 0}=\bar{x}_{11}-\bar{x}_{01}-\bar{x}_{10}+\bar{x}_{00}=\bar{x}_{1 \beta}-\bar{x}_{0 \beta}=\bar{x}_{\alpha \beta}
$$

and

$$
\Delta \bar{x}_{\alpha \frac{1}{2}}=\Delta \bar{x}_{1 \frac{1}{2}}-\Delta \bar{x}_{0 \frac{1}{2}} ; \quad \Delta \bar{x}_{\frac{1}{2} \beta}=\Delta \bar{x}_{\frac{1}{2} 1}-\Delta \bar{x}_{\frac{1}{2} 0} .
$$

Let $J(r, s)$ denote the Jacobian of $T$. Using the above notation, $J(r, s)$ can be written in the form

$$
J(r, s)=k_{0} s^{3}+A(r) s^{2}+B(r) s+C(r),
$$

where

$$
\begin{gathered}
k_{0}=8\left\{\Delta x_{1 \frac{1}{2}} \Delta y_{0 \frac{1}{2}}-\Delta x_{0 \frac{1}{2}} \Delta y_{1 \frac{1}{2}}\right\} \\
A(r)=12\left(\Delta x_{\frac{1}{2} \beta} \Delta y_{\alpha \frac{1}{2}}-\Delta x_{\alpha \frac{1}{2}} \Delta y_{\frac{1}{2} \beta}\right) r^{2} \\
+2\left\{\left(x_{\alpha \beta} \Delta y_{\alpha \frac{1}{2}}-\Delta x_{\alpha \frac{1}{2}} y_{\alpha \beta}\right)+2\left(\Delta x_{\alpha \frac{1}{2}} \Delta y_{\frac{1}{2} \beta}-\Delta x_{\frac{1}{2} \beta} \Delta y_{\alpha \frac{1}{2}}\right)\right. \\
\left.+8\left(\Delta x_{\frac{1}{2} \beta} \Delta y_{0 \frac{1}{2}}-\Delta y_{\frac{1}{2} \beta} \Delta x_{0 \frac{1}{2}}\right)\right\} r \\
+2\left\{\left(\Delta x_{\alpha \frac{1}{2}} y_{0 \beta}-x_{0 \beta} \Delta y_{\alpha \frac{1}{2}}\right)+2\left(x_{\alpha \beta} \Delta y_{0 \frac{1}{2}}-\Delta x_{0 \frac{1}{2}} y_{\alpha \beta}\right)\right. \\
\left.+4\left(\Delta x_{0 \frac{1}{2}} \Delta y_{\frac{1}{2} \beta}-\Delta x_{\frac{1}{2} \beta} \Delta y_{0 \frac{1}{2}}\right)+6\left(\Delta x_{0 \frac{1}{2}} \Delta y_{1 \frac{1}{2}}-\Delta x_{1 \frac{1}{2}} \Delta y_{0 \frac{1}{2}}\right)\right\},
\end{gathered}
$$




$$
\begin{aligned}
& B(r)=2\left\{\left(\Delta x_{\frac{1}{2} \beta} y_{\alpha \beta}-x_{\alpha \beta} \Delta y_{\frac{1}{2} \beta}\right)+2\left(\Delta x_{\alpha \frac{1}{2}} \Delta y_{\frac{1}{2} \beta}-\Delta x_{\frac{1}{2} \beta} \Delta y_{\alpha \frac{1}{2}}\right)\right. \\
& \left.+8\left(\Delta x_{\frac{1}{2} 0} \Delta y_{\alpha \frac{1}{2}}-\Delta x_{\alpha \frac{1}{2}} \Delta y_{\frac{1}{2} 0}\right)\right\} r^{2} \\
& +4\left\{\left(\Delta x_{\frac{1}{2} \beta} y_{0 \beta}-x_{0 \beta} \Delta y_{\frac{1}{2} \beta}\right)+\left(x_{\alpha 0} \Delta y_{\alpha \frac{1}{2}}-\Delta x_{\alpha \frac{1}{2}} y_{\alpha 0}\right)\right. \\
& +2\left\{\left(\Delta x_{0 \frac{1}{2}} \Delta y_{\frac{1}{2} 1}-\Delta x_{\frac{1}{2} 1} \Delta y_{0 \frac{1}{2}}\right)+\left(\Delta x_{1 \frac{1}{2}} \Delta y_{\frac{1}{2} 0}-\Delta x_{\frac{1}{2} 0} \Delta y_{1 \frac{1}{2}}\right)\right. \\
& \left.+8\left(\Delta x_{\frac{1}{2} 0} \Delta y_{0 \frac{1}{2}}-\Delta x_{0 \frac{1}{2}} \Delta y_{\frac{1}{2} 0}\right)\right\} r \\
& +\left\{x_{\alpha \beta} y_{0 \beta}-x_{0 \beta} y_{\alpha \beta}+2\left\{x_{0 \beta}\left(\Delta y_{\alpha \frac{1}{2}}+\Delta y_{\frac{1}{2} \beta}\right)-\left(\Delta x_{\alpha \frac{1}{2}}+\Delta x_{\frac{1}{2} \beta}\right) y_{0 \beta}\right.\right. \\
& \left.+\Delta x_{0 \frac{1}{2}} y_{\alpha \beta}-x_{\alpha \beta} \Delta y_{0 \frac{1}{2}}\right\} \\
& +4\left\{\left(\Delta x_{1 \frac{1}{2}} \Delta y_{0 \frac{1}{2}}-\Delta x_{0 \frac{1}{2}} \Delta y_{1 \frac{1}{2}}\right)\right. \\
& \left.+\left(\Delta x_{\frac{1}{2} 1} \Delta y_{0 \frac{1}{2}}-\Delta x_{0 \frac{1}{2}} \Delta y_{\frac{1}{2} 1}\right)+\left(x_{\alpha 0} \Delta y_{0 \frac{1}{2}}-\Delta x_{0 \frac{1}{2}} y_{\alpha 0}\right)\right\} \\
& \left.+12\left(\Delta x_{0 \frac{1}{2}} \Delta y_{\frac{1}{2} 0}-\Delta x_{\frac{1}{2} 0} \Delta y_{0 \frac{1}{2}}\right)\right\} \text {, }
\end{aligned}
$$

and

$$
\begin{aligned}
& C(r)=8\left\{\Delta x_{\frac{1}{2} 0} \Delta y_{\frac{1}{2} 1}-\Delta x_{\frac{1}{2} 1} \Delta y_{\frac{1}{2} 0}\right\} r^{3} \\
& +2\left\{\left(x_{\alpha 0} \Delta y_{\frac{1}{2} \beta}-\Delta x_{\frac{1}{2} \beta} y_{\alpha 0}\right)+2\left(\Delta x_{\frac{1}{2} 0} y_{\alpha \beta}-x_{\alpha \beta} \Delta y_{\frac{1}{2} 0}\right)\right. \\
& \left.+4\left(\Delta x_{\alpha \frac{1}{2}} \Delta y_{\frac{1}{2} 0}-\Delta x_{\frac{1}{2} 0} \Delta y_{\alpha \frac{1}{2}}\right)+6\left(\Delta x_{\frac{1}{2} 1} \Delta y_{\frac{1}{2} 0}-\Delta x_{\frac{1}{2} 0} \Delta y_{\frac{1}{2} 1}\right)\right\} r^{2} \\
& +\left\{\left(x_{\alpha 0} y_{\alpha \beta}-x_{\alpha \beta} y_{\alpha 0}\right)+2\left\{\left(\Delta x_{\frac{1}{2} \beta}+x_{\alpha \frac{1}{2}}\right) y_{\alpha 0}-x_{\alpha 0}\left(\Delta y_{\frac{1}{2} \beta}+\Delta y_{\alpha \frac{1}{2}}\right)\right.\right. \\
& \left.+\left(x_{\alpha \beta} \Delta y_{\frac{1}{2} 0}-\Delta x_{\frac{1}{2} \alpha} y_{\alpha \beta}\right)\right\} \\
& +4\left\{\left(\Delta x_{\frac{1}{2} 0} \Delta y_{\frac{1}{2} 1}-\Delta x_{\frac{1}{2} 1} \Delta y_{\frac{1}{2} 0}\right)\right. \\
& \left.+\left(\Delta x_{\frac{1}{2} 0} \Delta y_{1 \frac{1}{2}}-\Delta x_{1 \frac{1}{2}} \Delta y_{\frac{1}{2} 0}\right)+\left(\Delta x_{\frac{1}{2} \mathrm{O}} y_{0 \beta}-x_{0 \beta} \Delta y_{\frac{1}{2} \mathrm{o}}\right)\right\} \\
& \left.+12\left\{\Delta x_{0 \frac{1}{2}} \Delta y_{\frac{1}{2} 0}-\Delta x_{\frac{1}{2} 0} \Delta y_{0 \frac{1}{2}}\right\}\right\} r \\
& +\left\{x_{\alpha 0} y_{0 \beta}-x_{0 \beta} y_{\alpha 0}\right. \\
& +2\left\{\left(\Delta x_{0 \frac{1}{2}} y_{\alpha 0}-x_{\alpha 0} \Delta y_{0 \frac{1}{2}}\right)+\left(x_{0 \beta} \Delta y_{\frac{1}{2} 0}-\Delta x_{\frac{1}{2} 0} y_{0 \beta}\right)\right\} \\
& \left.+4\left(\Delta x_{\frac{1}{2} 0} \Delta y_{0 \frac{1}{2}}-\Delta x_{0 \frac{1}{2}} \Delta y_{\frac{1}{2} 0}\right)\right\} \text {. }
\end{aligned}
$$

An equivalent expression for $J(r, s)$ is

$$
J(r, s)=\kappa_{0} r^{3}+\alpha(s) r^{2}+\beta(s) r+\gamma(s),
$$

where $\gamma_{0}, \alpha(\cdot), \beta(\cdot)$, and $\gamma(\cdot)$ are obtained from $k_{0}, A(\cdot), B(\cdot)$, and $C(\cdot)$ by replacing $x_{i j}$ with $y_{j i}$ and $y_{i j}$ with $x_{j i}$, respectively.

Since $J(r, s)$ is continuous on $U, J(r, s)$ must attain a minimum value and a maximum value on $U$. It will be helpful to establish the sign of these values of $J(r, s)$ whenever these values occur in the interior of $U$, int $(U)$. The proof of the following theorem will be used in locating local extrema in $U$. 
THEOREM 2.2. Let $J(r, s)$ denote the Jacobian of the 8-node quadratic isoparametric transformation defined in (2.1). If both of the polynomials $D(r)$ and $\delta(s)$ in (2.6) and (2.7) are not identically zero, then the critical points of $J(r, s)$ on $U$ are isolated.

Proof. By the definition of a critical point of $J(r, s)$ at $\left(r^{*}, s^{*}\right)$,

$$
\left.\frac{\partial J}{\partial r}\right|_{\left(r^{*}, s^{*}\right)}=\left.\frac{\partial J}{\partial s}\right|_{\left(r^{*}, s^{*}\right)}=0
$$

Thus $\left(r^{*}, s^{*}\right)$ must satisfy

$$
\frac{\partial J}{\partial r} \equiv F(r, s)=A^{\prime}(r) s^{2}+B^{\prime}(r) s+C^{\prime}(r)=0,
$$

and

$$
\frac{\partial J}{\partial s} \equiv G(r, s)=3 k_{0} s^{2}+2 A(r) s+B(r)=0 .
$$

The hypothesis will ensure that (2.4) has a finite number of roots. By considering $F(r, s)$ and $G(r, s)$ as polynomials in $s$ with variable coefficients in $r$, a resultant of (2.4) is a polynomial in $r$. This is seen as follows.

A resultant of two polynomials is a polynomial whose coefficients depend upon the coefficients of the two given polynomials and becomes the zero polynomial if and only if the two given polynomials have a common nonconstant factor. Such a polynomial, having only the constant term, is called the "ordinary" resultant. The construction of resultants by means of bigradients is shown in [3].

For two polynomials

$$
f(x)=a_{0} x^{n}+a_{1} x^{n-1}+\cdots+a_{n}
$$

and

$$
g(x)=b_{0} x^{m}+b_{1} x^{m-1}+\cdots+b_{m},
$$

the ordinary resultant is given by

$$
\operatorname{det}\left|\begin{array}{cccccc}
a_{0} & a_{1} & & \cdots & & a_{m+n-1} \\
0 & a_{0} & & \cdots & & a_{m+n-2} \\
\vdots & \vdots & & & & \vdots \\
0 & 0 & \ldots & a_{0} & \ldots & a_{n} \\
0 & 0 & \ldots & b_{0} & \ldots & b_{m} \\
\vdots & \vdots & & & & \vdots \\
0 & b_{0} & & \ldots & & b_{m+n-2} \\
b_{0} & b_{1} & & \cdots & & b_{m+n-1}
\end{array}\right|,
$$

where the first $m$ rows are made up from the coefficients of $f(x)$, the last $n$ rows are made up from the coefficients of $g(x), a_{k}=0$ for $k>n$, and $b_{j}=0$ for $j>m$.

By combining (2.5) with (2.4), (2.5) can be written as the following polynomial in $r$ :

$$
D(r)=\operatorname{det}\left|\begin{array}{cccc}
A^{\prime}(r) & B^{\prime}(r) & C^{\prime}(r) & 0 \\
0 & A^{\prime}(r) & B^{\prime}(r) & C^{\prime}(r) \\
0 & 3 k_{0} & 2 A(r) & B(r) \\
3 k_{0} & 2 A(r) & B(r) & 0
\end{array}\right| .
$$


Therefore, from the definitions of the ordinary resultant and of critical points, the critical points of $J(r, s)$ occur in $U$ if and only if there exists $\left(r^{*}, s^{*}\right) \in U$ such that $D\left(r^{*}\right)=0$ and both $F\left(r^{*}, s^{*}\right)=G\left(r^{*}, s^{*}\right)=0$. Since $D(r)$ is a polynomial of at most 7 degrees, $D(r)$ will have at most 7 real roots since $D(r)$ is not the zero polynomial by hypothesis. By the definition of the resultant, for each of these roots, there exists at least one $s^{*}$, such that (2.4) is satisfied.

Similarly, considering $F(r, s)$ and $G(r, s)$ as polynomials in $r$ with variable coefficients in $s$ yields

$$
\delta(s) \equiv \operatorname{det}\left|\begin{array}{cccc}
3 \kappa_{0} & 2 \alpha(s) & \beta(s) & 0 \\
0 & 3 \kappa_{0} & 2 \alpha(s) & \beta(s) \\
0 & \alpha^{\prime}(s) & \beta^{\prime}(s) & \gamma^{\prime}(s) \\
\alpha^{\prime}(s) & \beta^{\prime}(s) & \gamma^{\prime}(s) & 0
\end{array}\right| .
$$

Since $\delta(s)$ is also not identically zero by hypothesis, there can be at most finitely many pairs $(r, s)$ satisfying $(2.4)$.

Remarks. Bezout's Theorem [5, Section 83] states that (2.4) can have no more than four isolated roots. Inasmuch as Theorem 2.2 produces an upper bound of 49 isolated roots, the main intent of Theorem 2.2 is to recognize when isolated roots occur as well as providing a means to find the isolated roots when they lie in $U$. It may not be necessary to actually solve $D(r)$ and $\delta(s)$ since Sturm sequences can determine the number of roots of $D(r)$ and $\delta(s)$ in $[0,1]$. Rather than using a general code which searches and finds all isolated roots of (2.4), a combination of Sturm sequences and bisections would be appropriate in confining a search to $U$.

Notice that $\delta(s)$ in (2.7) reducing to the zero polynomial, $\delta(s) \equiv 0$, implies that, for each real number $s$, there exists an $r$, possibly complex, such that (2.4) is satisfied. Further, the roots of (2.4) may no longer be isolated. In fact for fixed $s$, $J(r, s)$ could be a constant. However, provided $D(r) \neq 0$ and $k_{0} \neq 0$ when $\delta(s) \equiv$ 0 , the extrema of $J(r, s)$ remain isolated.

Theorem 2.3. If in Theorem 2.2, $D(r) \neq 0$, and $k_{0} \neq 0\left(\right.$ or,$\delta(s) \neq 0$ and $\left.\kappa_{0} \neq 0\right)$, then the critical points of $J(r, s)$ are isolated.

Proof. In (2.4) $k_{0} \neq 0$ implies that $G(r, s)$ is quadratic in $s$ with nonzero leading coefficient, and therefore, for each fixed $r, G(r, s)$ has at most two roots. $D(r) \neq 0$, implies that $D(r)$ has at most seven real roots. Hence there can be at most 14 pairs of $\left(r^{*}, s^{*}\right)$ coordinates which satisfy $G(r, s)=0$. The critical points of $J(r, s)$ must then be isolated.

When $k_{0}=0, G(r, s)$ in (2.4) is linear in $s$. By solving for $s$ in $G(r, s)=0$, $s \equiv s(r)$, then $F(r, s(r))=0$ reduces to solving a polynomial equation in $r$. If $F(r, s(r)) \equiv 0$, i.e. reduces to the zero polynomial, then the locus of critical points of $J(r, s)$ is a curve $(r,-B(r) / 2 A(r))$, and $J(r, s)$ is a constant on that curve. That $J(r, s)$ is constant on that curve follows from

$$
\frac{d}{d r} J(r, s(r))=\frac{\partial J}{\partial r}+\frac{\partial J}{\partial s} \frac{d s}{d r}=0 .
$$

Whether or not the curve of critical points intersects $U, J(r, s)$ will not vanish on $U$ as long as $J(r, s)$ does not vanish on the boundary of $U$. Similar conclusions pertain to the case of $\kappa_{0}=0$ and $G(s, r(s)) \equiv 0$. The following theorem includes the above remarks and also applies to the case where $k_{0}=0$ and $F(r, s(r)) \neq 0$. 
THEOREM 2.4. Let $J(r, s)$ in (2.2) and (2.3) denote the Jacobian of the isoparametric transformation defined in (2.1). Let the polynomials $J(0, s), J(1, s), J(r, 0)$ and $J(r, 1)$ be positive (negative) on $[0,1]$.

(1) If $k_{0}=0$, then $J(r, s)>0(<0),(r, s) \in U$, if and only if $\left(r^{*}, s^{*}\right) \in U$ satisfying

$$
A^{\prime}(r)\{B(r)\}^{2}-2 A(r) B(r) B^{\prime}(r)+4\{A(r)\}^{2} C^{\prime}(r)=0
$$

and

$$
2 A(r) s+B(r)=0
$$

implies $J\left(r^{*}, s^{*}\right)>0(<0)$.

(2) If $\kappa_{0}=0$, then $J(r, s)>0(<0),(r, s) \in U$, if and only if $\left(r^{*}, s^{*}\right) \in U$ satisfying

$$
\alpha^{\prime}(s)\{\beta(s)\}^{2}-2 \alpha(s) \beta(s) \beta^{\prime}(s)+4\{\alpha(s)\}^{2} \gamma^{\prime}(s)=0
$$

and

$$
2 \alpha(s) r+\beta(s)=0
$$

implies $J\left(r^{*}, s^{*}\right)>0(<0)$.

Proof. By symmetry, it suffices to consider the case $k_{0}=0$ and $J(r, s)>0$ on the boundary of $U$. For $r^{*} \in(0,1)$ and $A\left(r^{*}\right) \neq 0, G\left(r^{*}, s\right)$ is linear in $s$. Back substitution in (2.4) yields the desired polynomial in $(2.8 \mathrm{a})$. For $r^{*} \in(0,1)$ such that $A\left(r^{*}\right)=0$, by the expression for $G\left(r^{*}, s\right)$ in $(2.4), B\left(r^{*}\right)=0$, when $\left(r^{*}, s^{*}\right)$ is a critical point. But $A\left(r^{*}\right)=B\left(r^{*}\right)=k_{0}=0$ implies $J\left(r^{*}, s^{*}\right)=J\left(r^{*}, 0\right)>0$ by the representation of $J\left(r^{*}, s^{*}\right)$ in (2.2) and the hypothesis that $J(r, s)>0$ on the boundary of $U$. If $(2.8 \mathrm{a})$ is identically zero and the curve of critical points $(r,-B(r) / 2 A(r))$ intersects $U$, by hypothesis and that $J(r,-B(r) / 2 A(r))$ is constant, $J(r,-B(r) / 2 A(r))>0$ and Theorem 2.3 is proved.

Remarks. In Theorem 2.4, verifying the hypothesis that $J(0, s), J(1, s), J(r, 0)$, $J(r, 1)$ are positive (or negative) on $[0,1]$ is a simple task since $J(0, s), J(1, s)$, $J(r, 0), J(r, 1)$ are at most cubic polynomials. To show that any cubic polynomial $P(x)$ does not change sign on $[0,1]$ is numerically straightforward. Show that $P(0) P(1)>0$ and apply the quadratic formula to show that either $P^{\prime}(x)$ has no roots in $(0,1)$ or, when $P^{\prime}(c)=0$ for $c \in(0,1)$, that $P(0) P(c)>0$.

An algorithm presented in flow chart form in Figure 2.1 will determine whether the Jacobian of any transformation defined in (2.1) will vanish. For those transformations where $(2.2)$ reduces to

$$
J(r, s)=a_{11} r s+a_{10} r+a_{01} s+a_{00},
$$

no change in sign in the set $\{J(0,0), J(1,0), J(0,1), J(1,1)\}$ implies that $J(r, s)$ is of the same sign throughout $U$. The only transformations for which the nonvanishing of its Jacobian cannot be determined by the above theorems and remarks are those transformations for which $k_{0} \kappa_{0} \neq 0$ and both $D(r) \equiv 0$ and $\delta(s) \equiv 0$. The following lemmas consider such transformations.

LEMMA 2.5. Let $J(r, s)$ be the Jacobian of the isoparametric transformation given in (2.1). If in (2.6) and (2.7) both $D(r) \equiv 0$ and $\delta(s) \equiv 0$ and $k_{0} \kappa_{0} \neq 0$, then $A(r)$ in (2.2) and $\alpha(s)$ in (2.3) are linear polynomials. 


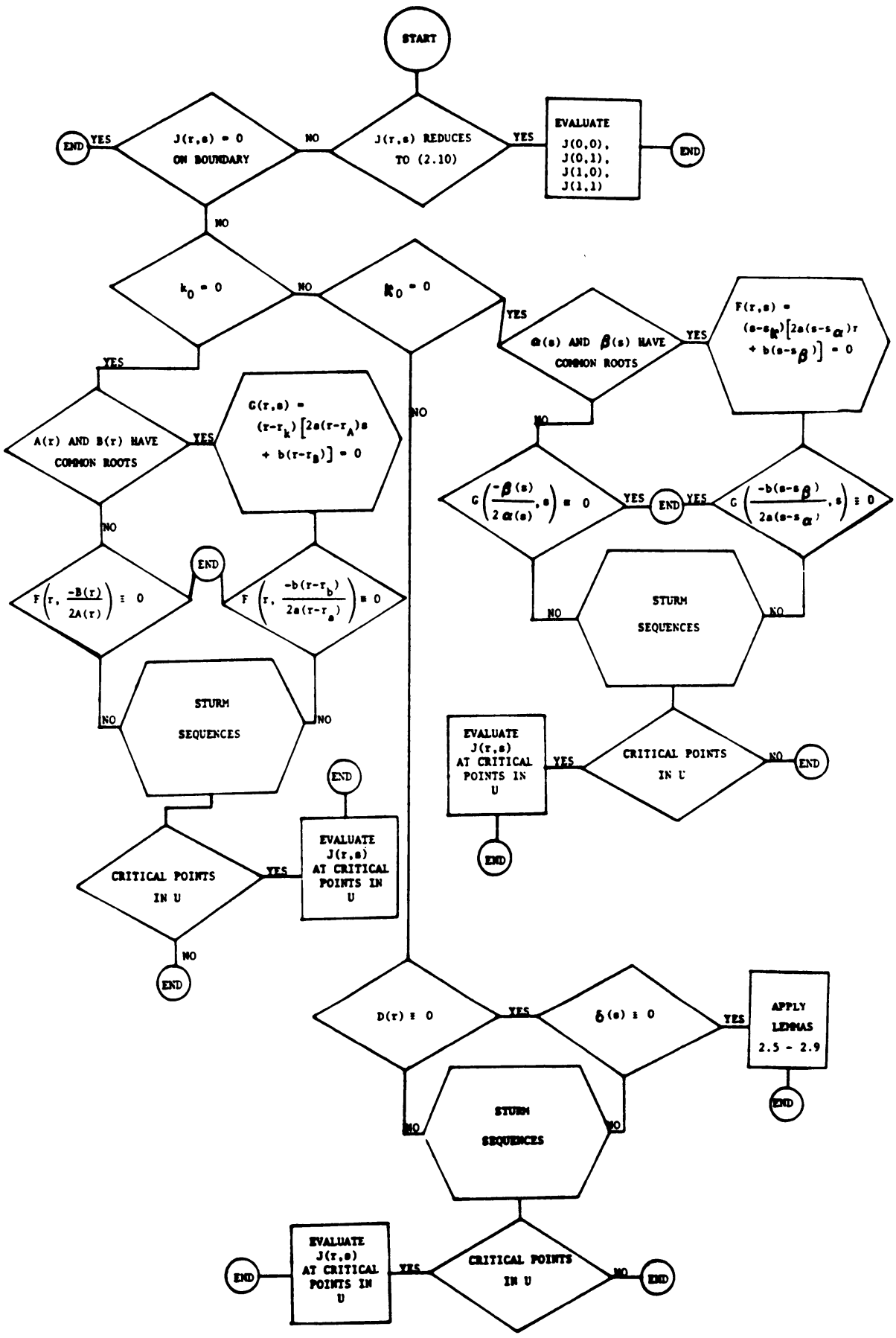

FIGURE 2.1

An algorithm, based on the theorems in Section 2, for identifying nonvanishing Jacobians of the isoparametric transformations in (2.1) 
Proof. The leading coefficient of $D(r)$ in (2.7) is given by the leading coefficient of the product $12 \kappa_{0} A^{\prime}(r) A(r)^{2} C^{\prime}(r)$. Since $D(r) \equiv 0$ and $\kappa_{0} k_{0} \neq 0$, the leading coefficient of $A(r)$ must be zero. Consequently $A(r)$ is linear. Similarly $\alpha(s)$ is linear.

LEMMA 2.6. If $A(r)$ is a constant, the extrema $\left(r^{*}, s^{*}\right)$ of $J(r, s)$ are of the form $\left(r^{*}, s\left(r^{*}\right)\right)$, where $s(r)=-C^{\prime}(r) / B^{\prime}(r)$ and $r^{*}$ satisfies

$$
\left[3 k_{0} C^{\prime}(r)-2 A(r) B^{\prime}(r)\right] C^{\prime}(r)+\left[B^{\prime}(r)\right]^{2} B(r)=0 .
$$

Proof. Suppose $A(r)$ is a constant. Solving for $s$ in $F(r, s)=0$, yields $s(r)$. Substituting $s(r)$ into $G(r, s)$ yields (2.11).

When (2.11) is satisfied for all $r^{*}, 0 \leqslant r^{*} \leqslant 1, J(r, s)$ is constant on the locus of extrema of the form $(r, s(r))$. It then suffices to evaluate $J(r, s)$ on the boundary of $U$. On the other hand when (2.11) is not identically zero, (2.11) yields at most four solutions $r^{*}, 0 \leqslant r^{*} \leqslant 1$ so that $J(r, s)$ can have at most four extrema in $U$ of the form $\left(r^{*},-C^{\prime}\left(r^{*}\right) / B\left(r^{*}\right)\right)$.

Based upon Lemmas 2.5 and 2.6 assume that $A(r)$ is linear but not a constant and proceed by expressing $D(r)$ as follows:

$$
D(r)=f(r) h(r)-[g(r)]^{2},
$$

where

$$
f(r)=3 k_{0} B^{\prime}(r)-2 A(r) A^{\prime}(r), \quad g(r)=3 k_{0} C^{\prime}(r)-B(r) A^{\prime}(r),
$$

and

$$
h(r)=2 A(r) C^{\prime}(r)-B(r) B^{\prime}(r) .
$$

LEMMA 2.7. Let $D(r) \equiv 0, k_{0} \kappa_{0} \neq 0$ and $A(r)$ not be a constant. If $f(r) \equiv 0$, then the extrema $\left(r^{*}, s^{*}\right)$ of $J(r, s)$ are of the form $\left(r^{*}, s\left(r^{*}\right)\right)$, where

$$
s(r)=\left(-A(r) \pm\left\{[A(0)]^{2}-3 k_{0} B(0)\right\}^{1 / 2}\right) / 3 k_{0}
$$

and $r^{*}$ satisfies

$$
A^{\prime}(r) s(r)^{2}+B^{\prime}(r) s(r)+C^{\prime}(r)=0 .
$$

Proof. $f(r) \equiv 0$ implies

$$
0=\int_{0}^{r} f(r) d r=3 k_{0} B(r)-[A(r)]^{2}-3 k_{0} B(0)+[A(0)]^{2} .
$$

Solving for $B(r)$ in the above expression and substitution into $G(r, s)$ yields

$$
\left[3 k_{0} s+A(r)\right]^{2}=[A(0)]^{2}-3 k_{0} B(0) .
$$

Consequently, solving for $s$ and substitution into $F(r, s)$ produces the polynomial equation in (2.13) with $s(r)$ given in (2.12).

Again, if (2.13) is identically zero, it suffices to evaluate $J(r, s)$ on the boundary of $U$.

LEMMA 2.8. Let $D(r) \equiv 0, k_{0} \kappa_{0} \neq 0$ and $A(r)$ not be a constant. If $h(r) \equiv 0$, then the extrema $\left(r^{*}, s^{*}\right)$ of $J(r, s)$ must satisfy either (2.12) and (2.13) or $r^{*}$ satisfies $f(r)=0$ and $s^{*}$ satisfies $G\left(r^{*}, s\right)=0$. 
Proof. $h(r) \equiv 0$ and $D(r) \equiv 0$ imply that $g(r) \equiv 0$. By eliminating $s^{2}$ in (2.4), an extremum $\left(r^{*}, s^{*}\right)$ of $J(r, s)$ must satisfy

$$
f(r) s+g(r)=0
$$

But $g(r) \equiv 0$ implies either $f(r) \equiv 0$, in which case Lemma 2.7 applies, or $f\left(r^{*}\right)=0$. In the latter case $f(r)$ is at most quadratic, yielding at most two roots which are substituted into $G(r, s)$ in (2.4).

LEMMA 2.9. Let $D(r) \equiv 0, k_{0} \kappa_{0} \neq 0$ and $A(r)$ not be a constant. If $g(r) \equiv 0$, then the extrema $\left(r^{*}, s^{*}\right)$ of $J(r, s)$ are either of the form $\left(r^{*}, s\left(r^{*}\right)\right)$ where $s(r)=$ $-g(r) / f(r), f\left(r^{*}\right) \neq 0$ and $r^{*}$ satisfies

$$
3 k_{0}[g(r)]^{2}-2 A(r) g(r) f(r)+B(r)[f(r)]^{2}=0
$$

or $\left(r^{*}, s^{*}\right)$ satisfies $f(r)=0$ and $G\left(r^{*}, s\right)=0$.

Proof. $g(r) \neq 0$ implies that $f(r) \neq 0$. An extremum $\left(r^{*}, s^{*}\right)$ must be such that $f\left(r^{*}\right)=0$ or $f\left(r^{*}\right) \neq 0$. If $f\left(r^{*}\right)=0$, the solution $r^{*}, 0<r^{*}<1$ of $f(r)=0$ is substituted into $G(r, s)=0$, producing at most four local extrema. If $f\left(r^{*}\right) \neq 0$, then as in (2.14) $s^{*}=g\left(r^{*}\right) / f\left(r^{*}\right)$ and $r^{*}$ must satisfy (2.15).

Again, when (2.15) is identically zero, $J(r, s)$ need only be evaluated on the boundary of $U$.

This section concludes by noting that Theorem 2.4 does not eliminate the possibility that isoparametric transformations such as those of Frey, Hall, and Porsching can have positive Jacobians on the boundary of $U$ and a negative Jacobian somewhere in the interior of $U$.

3. Sufficient Conditions for Invertibility. The theorems in this section give sufficient conditions for nonvanishing Jacobians. Thus, when a vanishing Jacobian is found by the algorithm in Figure 2.1, the transformation can be made invertible by redeploying the nodes to satisfy any one of several inequalities found in Theorem 3.2. These simple inequalities are easily utilized numerically and also provide a series of quick tests which will detect many transformations with nonvanishing Jacobians.

The theorems in this section are independent from the results in Section 2. The first theorem concerns semiquadrilaterals for which the semirectangle in $[1, p .728]$ is a special case. The main theorem applies to the arbitrary placement of the 8-nodes of the quadratic isoparametric element.

A semiquadrilateral is the image of an isoparametric transformation $T$ defined in (2.1), where $T(\partial U)=\{T(r, s):(r, s) \in \partial U\}$ is composed of three straight edges and one curved edge, see Figure 3.1.

In Figure 3.1,

$$
\bar{x}_{\frac{1}{2} 0}=\frac{\bar{x}_{00}+\bar{x}_{10}}{2}, \quad \bar{x}_{\frac{1}{2} 1}=\frac{\bar{x}_{01}+\bar{x}_{11}}{2}, \quad \text { and } \quad \bar{x}_{0 \frac{1}{2}}=\frac{\bar{x}_{00}+\bar{x}_{01}}{2} .
$$

(3.1) implies

$$
\Delta \bar{x}_{\frac{1}{2} 0}=\Delta \bar{x}_{\frac{1}{2} 1}=\Delta \bar{x}_{0 \frac{1}{2}}=\left(\begin{array}{l}
0 \\
0
\end{array}\right) .
$$

It then follows from (3.1), (3.2), and (2.3) that $J(r, s)$ reduces to

$$
J(r, s)=\beta(s) r+\gamma(s) .
$$




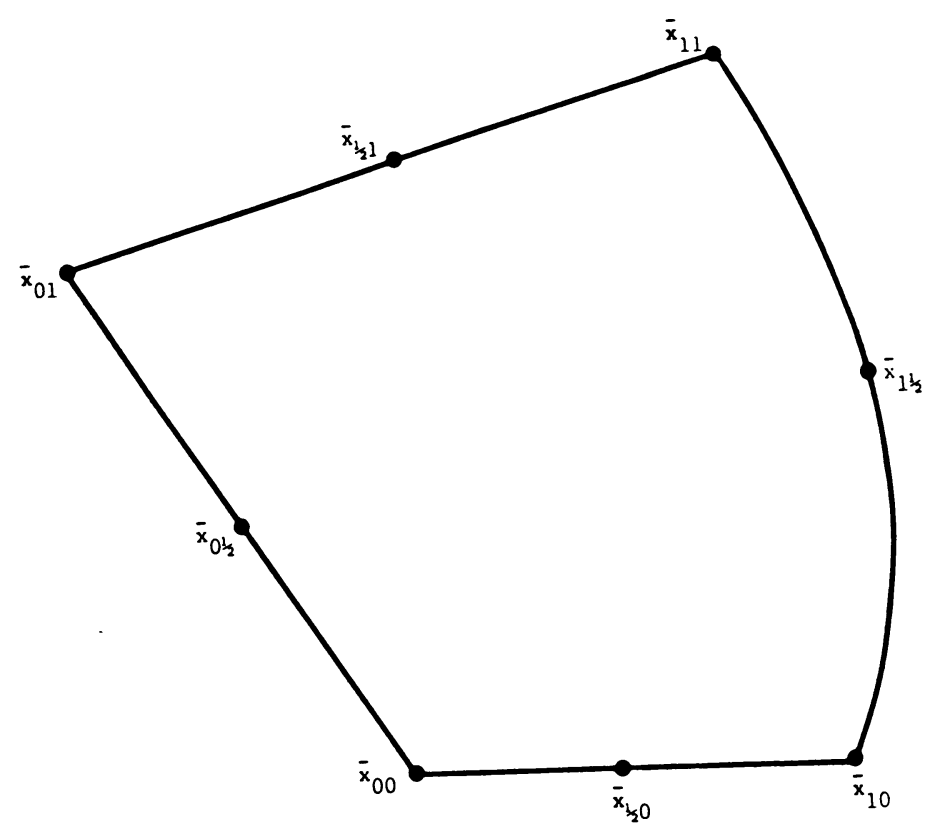

FIGURE 3.1

A semiquadrilateral

THEOREM 3.1. Let $J(r, s)$ be the Jacobian of the isoparametric transformation given in (2.1). If the image of $T$ is a semiquadrilateral satisfying (3.1), then $J(r, s)>0$ if and only if $J(r, s)>0$ on the boundary of $U$.

Proof. It follows from (3.1) and (2.3) that (3.2) and (3.3) hold. From (3.3) and $J(r, s)>0$ on the boundary of $U, J(0, s)>0$ implies $\gamma(s)>0,0<s<1$, and $J(1, s)>1$ implies $\beta(s)+\gamma(s)>0,0<s<1$. Because of the linearity in $r$ in (3.3), $J(r, s)>0$ for all $(r, s) \in U$.

THEOREM 3.2. Let $J(r, s)$ in $(2.2)$ and $(2.3)$ be the Jacobian of the isoparametric transformation in (2.1) and let $J(r, s)>0$ on the boundary of $U$.

A. If $3 k_{0}+A(r) \leqslant 0,0<r<1$, then $J(r, s)>0$ on $U$.

B. If $3 k_{0}+A(r)>0$ and $3 k_{0}+2 A(r)+B(r)<0,0<r<1$, then $J(r, s)>0$ on $U$.

C. If $k_{0}<0$, then each of the following conditions imply $J(r, s)>0$ on $U$ :

I. $B(r)+C(r)>0,0<r<1$. $0\}$.

II. $\phi=\left\{r \in(0,1): 3 k_{0}+2 A(r)+B(r)>0\right\} \cap\{r \in(0,1): B(r)+C(r)<$

D. If $k_{0}>0$, then each of the following conditions imply $J(r, s)>0$ on $U$ :

I. $A(r) \geqslant 0$ and $B(r)>0,0<r<1$.

II. $A(r)>0, B(r)<0$, and $B(r)+C(r)>0,0<r<1$.

The above statement may be written with $k_{0}, A(r), B(r)$, and $C(r)$ being replaced by $\gamma_{0}, \alpha(s), \beta(s)$, and $\gamma(s)$, respectively. 
Proof. This proof treats $J(r, s)$ as a surface over $U$ where $J(r, s)>0$ on the boundary of $U$. Also, when $r$ is fixed, $J(r, s)$ is a polynomial in $s$. By assumption, $J(r, 0)>0$ on $U$ implies $C(r)>0,0 \leqslant r \leqslant 1$. Thus, for each $r^{*}, 0 \leqslant r^{*} \leqslant 1$, $J\left(r^{*}, s\right)$ is a cubic (quadratic when $k_{0}=0$ ) polynomial in $s$ with constant positive coefficient $C\left(r^{*}\right)$.

Proof of part A. By assumption $J\left(r^{*}, 1\right)>0$ and $J\left(r^{*}, 0\right)>0$. Therefore, as a consequence of Rolle's theorem, in order for $J\left(r^{*}, s\right)$ to possess a root in $(0,1)$ there must exist $s^{*} \in(0,1)$ such that

$$
3 k_{0} s^{2}+2 A\left(r^{*}\right) s^{*}+B\left(r^{*}\right)=0 .
$$

Furthermore, $J\left(r^{*}, 1\right)>0$ and $J\left(r^{*}, 0\right)>0$ also imply that $J\left(r^{*}, s\right)$ has a local minimum at $s^{*}$, that is,

$$
6 k_{0} s^{*}+2 A\left(r^{*}\right)>0
$$

The hypothesis in part A ensures that (3.4) cannot be satisfied.

Proof of part B. Consider separately the cases $k_{0} \leqslant 0$ and $k_{0}>0$. For $k_{0} \leqslant 0$, write $J(r, s)$ in $(2.2)$ as

$$
\begin{aligned}
J(r, s)= & k_{0}(s-1)^{3}+\left(3 k_{0}+A(r)\right)(s-1)^{2}+\left(3 k_{0}+2 A(r)+B(r)\right)(s-1) \\
& +k_{0}+A(r)+B(r)+C(r) .
\end{aligned}
$$

In the above expession for $J(r, s)$, if for some $r^{*}$ the coefficients of $(s-1)^{k}$ alternate in sign, then $J\left(r^{*}, s\right)$ has no roots for $s-1 \leqslant 0 . k_{0} \leqslant 0$, condition II and $J(r, 1)>0$ ensure the alternation in sign for $0<r<1$. When $k_{0}>0$, for each fixed $r^{*}, J\left(r^{*}, s\right)$ is a cubic polynomial with a positive leading coefficient. Thus, if a local minimum of $J\left(r^{*}, s\right)$ exists at $s_{m}$, it is unique and $s_{M}<s_{m}$ where $J\left(r^{*}, s\right)$ has its unique local maximum at $s_{M}$. (Note that for $s$ negative and large in absolute value, $J\left(r^{*}, s\right)$ will be negative and increasing so that $s_{M}<s_{m}$.) Furthermore, $J\left(r^{*}, s\right)$ will be decreasing only when $s$ is in the interval $\left(s_{M}, s_{m}\right)$. Since $J\left(r^{*}, 0\right)>0$ and $J\left(r^{*}, 1\right)>0$ by hypothesis, it suffices to show that $J\left(r^{*}, s\right)$ is decreasing at $s=1$. That $d\left(J\left(r^{*}, s\right)\right) / d s<0$ at $s=1$ is condition II.

Proof of part C. Observe that $J\left(r^{*}, s\right)$ is at most a cubic polynomial with a nonpositive leading coefficient and such that $J\left(r^{*}, 0\right)>0$ and $J\left(r^{*}, 1\right)>0$. In condition $\mathrm{I}$, if $k_{0}=0$ and $B\left(r^{*}\right) \geqslant 0$, then $J\left(r^{*}, s\right)$ is a parabola in $s$ which is nondecreasing at $s=0$ and is positive at $s=0$ and $s=1$, implying that $J\left(r^{*}, s\right)>$ 0 for $0 \leqslant s \leqslant 1$. If $k_{0}<0$ and $B\left(r^{*}\right) \geqslant 0$, then $J\left(r^{*}, s\right)$ is nondecreasing at $J\left(r^{*}, 0\right)$ so that $J\left(r^{*}, s\right) \neq 0$ on $[0,1]$, otherwise $J\left(r^{*}, s\right)$ would have two inflection points on the interval $[0, \infty)$, which is impossible. If $B\left(r^{*}\right)<0$ and the tangent line $y=$ $B\left(r^{*}\right) s+C\left(r^{*}\right)$ to $J\left(r^{*}, s\right)$ at $s=0$ intersect the $s$-axis in the interval $[1, \infty)$, then $J\left(r^{*}, s\right) \neq 0$ on $[0,1]$ for otherwise $J\left(r^{*}, s\right)$ would have at least two inflection points which is again impossible for cubic polynomials. That the intersection of $y=$ $B\left(r^{*}\right) s+C\left(r^{*}\right)$ occurs in $[1, \infty)$ and can be written as $1<-C\left(r^{*}\right) / B\left(r^{*}\right)$ is condition I. Note that when $B\left(r^{*}\right) \geqslant 0$ condition I is also satisfied. Condition II is easily deduced from part $B$ and condition $I$ of part $C$.

Proof of part D. Condition I implies that the coefficients of $J\left(r^{*}, s\right)$ are all nonnegative for $0<r^{*}<1$, and hence $J\left(r^{*}, s\right)$ cannot be negative for $0<s<1$.

Finally, $A\left(r^{*}\right) \geqslant 0$ implies that the inflection point of $J\left(r^{*}, s\right)$ occurs for $s<0$ and that $J\left(r^{*}, s\right)$ is concave up on $[0,1]$. The equation of the tangent line to $J\left(r^{*}, s\right)$ 
at $s=0$ is $y=B\left(r^{*}\right) s+C\left(r^{*}\right)$. If $B\left(r^{*}\right) \leqslant 0$ and the tangent line intersects the $s$-axis in the interval $[1, \infty)$, then $J\left(r^{*}, s\right) \neq 0$ for $0<s<1$, otherwise $J\left(r^{*}, s\right)$ would have two inflection points. That $A\left(r^{*}\right) \geqslant 0, B\left(r^{*}\right) \leqslant 0$ and the intersection occurs at $s=-C\left(r^{*}\right) / B\left(r^{*}\right) \leqslant 0$ is condition III.

By changing the roles of $r$ and $s$, Theorem 3.2 is proven.

When $k_{0} \gamma_{0}=0$, Theorem 3.2 is much simplified and condition C.I. can be strengthened. It is of interest to note that the isoparametric transformations in [1] all have the constraint

$$
\begin{gathered}
x_{\frac{1}{2} 0}=\frac{x_{00}+x_{10}}{2}, \quad x_{\frac{1}{2} 1}=\frac{x_{01}+x_{11}}{2}, \quad y_{0 \frac{1}{2}}=\frac{y_{00}+y_{01}}{2}, \quad \text { and } \\
y_{1 \frac{1}{2}}=\frac{y_{10}+y_{11}}{2},
\end{gathered}
$$

which implies that both $k_{0}=0$ and $\kappa_{0}=0$. The following corollary which strengthens C.I. also shows that for transformations satisfying (3.5) it may not be true that a nonvanishing Jacobian on the boundary of $U$ implies the Jacobian is nonvanishing on the interior of $U$.

COROLlaRY 3.3. Let $T$ be an isoparametric finite element transformation defined in (2.1) such that $k_{0} \kappa_{0}=0$. A necessary condition for the existence of $\left(r^{*}, s^{*}\right) \in U$ for which $J\left(r^{*}, s^{*}\right)<0$ when $J(r, s)>0$ on the boundary of $U$ is that $\left(r^{*}, s^{*}\right)$ satisfy inequalities (1)-(4) when $k_{0}=0$ and $(1)^{\prime}-(4)^{\prime}$ when $\kappa_{0}=0$.
(1) $A(r)>0$,
(1) $\alpha(s)>0$,
(2) $2 A(r)+B(r)>0$,
$(2)^{\prime} 2 \alpha(s)+\beta(s)>0$,
(3) $B(r)<0$,
(3) $\beta(s)<0$,
(4) $B(r)+\sqrt{2} C(r)<0$,
(4) $\beta(s)+\sqrt{2} \gamma(s)<0$.

Proof. By symmetry it will suffice to consider only the inequalities involving $r$. $A\left(r^{*}\right)>0$, for otherwise by the proof of part A in Theorem 3.2, $J\left(r^{*}, s\right)>0$, on $[0,1]$. Consequently, $2 A\left(r^{*}\right)+B\left(r^{*}\right)>0$, for otherwise by the proof of part B in Theorem 3.2, $J\left(r^{*}, s\right)>0$ on $[0,1]$. Since $A\left(r^{*}\right)>0$, and $C\left(r^{*}\right)>0$ by hypothesis, $B\left(r^{*}\right)<0$, for otherwise all the coefficients of $J\left(r^{*}, s\right)$, as a polynomial in $s$, are positive implying $J\left(r^{*}, s\right)>0$ on $[0,1] . B\left(r^{*}\right)+C\left(r^{*}\right)<0$, for otherwise by the proof of part C condition $I, J\left(r^{*}, s\right)>0$ on $[0,1]$. Since $J\left(r^{*}, s\right)$ is a quadratic in $s$, $J\left(r^{*}, s^{*}\right)<0,0<s^{*}<1$, implies that the discriminant $B\left(r^{*}\right)^{2}-4 A\left(r^{*}\right) C\left(r^{*}\right)>0$. Combining (2) and $B\left(r^{*}\right)+C\left(r^{*}\right)<0$ implies $2 A\left(r^{*}\right)>C\left(r^{*}\right)=J\left(r^{*}, 0\right)>0$. Further, substitution into the discriminant yields $B\left(r^{*}\right)^{2}-2 C\left(r^{*}\right)^{2}>0$ or $\left(B\left(r^{*}\right)-\sqrt{2} C\left(r^{*}\right)\right)\left(B\left(r^{*}\right)+\sqrt{2} C(r)\right)>0$. Since $B\left(r^{*}\right)-\sqrt{2} C\left(r^{*}\right)<0$ inequality (4) must be satisfied and the corollary is proved.

The flow chart in Figure 3.1 indicates how Theorem 3.2 can be used to detect many quadratic isoparametric transformations with vanishing Jacobians. Since the algorithm involves only the sign behavior of at most cubic polynomials on $[0,1]$ the algorithm is computationally straightforward. Theorem 3.2 can be further utilized in rectifying finite elements, whose Jacobians do not vanish on the boundary of $U$ but vanish on the interior of $U$, by repositioning the nodes so that a quadratic inequality such as $3 k_{0}+A(r) \geqslant 0$ is satisfied. 


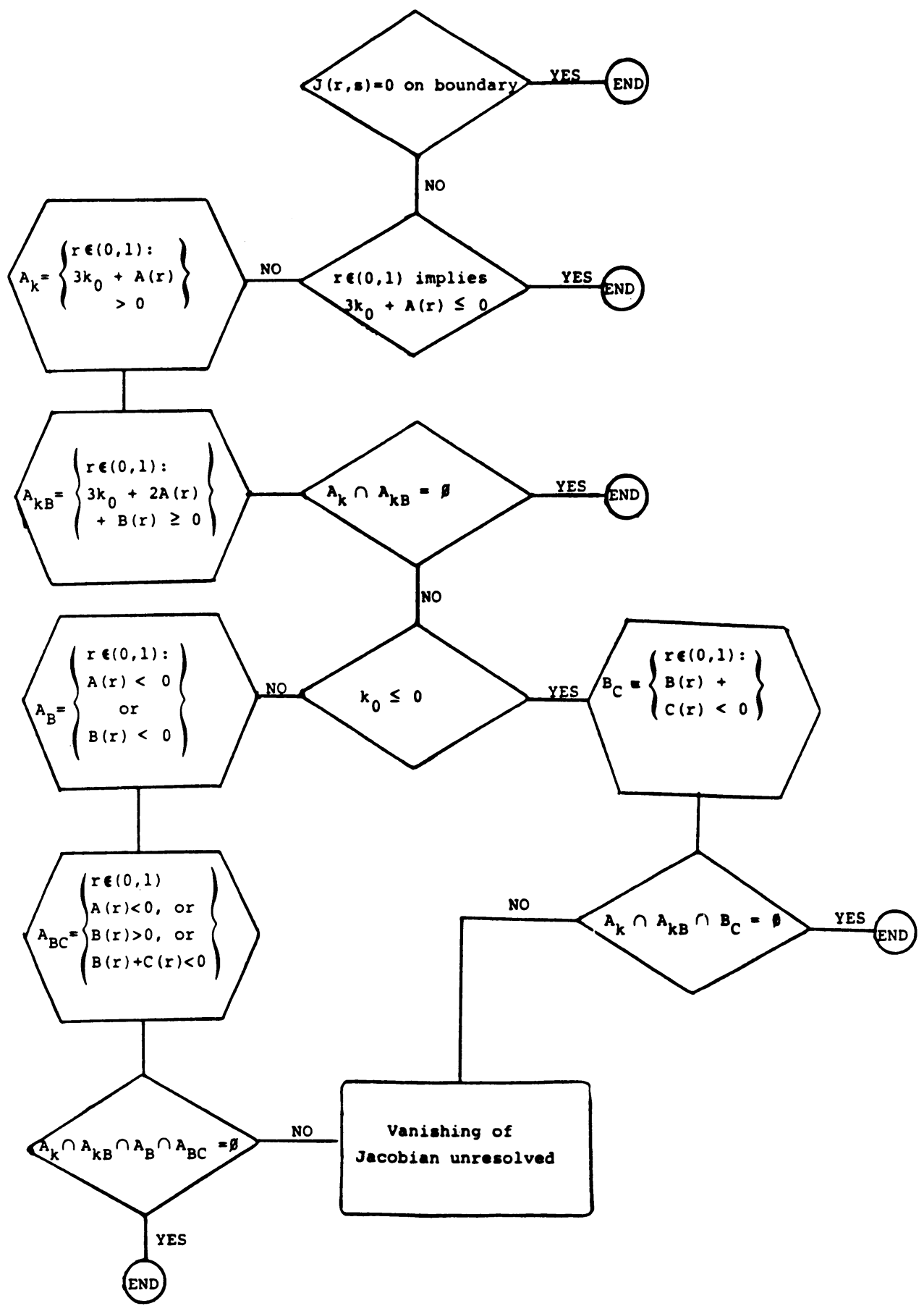

FIGURE 3.2

An algorithm, based on Theorem 3.2, for identifying nonvanishing Jacobians of transformations in (2.1) 
Acknowledgements. The author appreciates many conversations with Dr. James C. Cavendish concerning this paper.

Mathematics Department

General Motors Research Laboratories

Warren, Michigan 48090

1. A. E. Frey, C. A. Hall \& T. A. Porsching, "Some results on the global inversion of bilinear and quadratic isoparametric finite element transformations," Math. Comp., v. 32, 1978, pp. 725-749.

2. W. Fulks, Advanced Calculus, Wiley, New York, 1961.

3. A. S. Householder, "Bigradients and the problem of Routh and Hurwitz," SIAM Rev., v. 10, 1968, pp. 56-66.

4. C. J. De la Vallée Poussin, Cours d'Analyse Infinitésimale, Vol. I, 7th ed., Louvain, 1930.

5. B. L. VAN DER WAERDEN, Modern Algebra, Vol. II, Ungar, New York, 1950. 